\title{
Post-vaccination Tubercular Cold Abscess of Thigh of An Infant
}

\author{
Tabindah Jahan, Nahid Nehvi, Shariq Farooq \\ Department of Microbiology, Government Medical College, Srinagar
}

\begin{abstract}
We report a case of post immunization tubercular soft tissue cold abscess on a lateral part of the right thigh in an eight-month-old male baby following third dose of intramuscular Hepatitis B vaccination. The baby presented with gradual onset of fever for 10 days after hepatitis B vaccination this was followed by an erythematous, painless, firm small swelling on the anterolateral part of the right thigh which grew gradually to a size of $2 \times 2.5 \mathrm{~cm}$ size. The baby was treated with empirical antibiotics (Amoxicillin) following which fever subsided but there was no improvement in swelling so the baby was advised for various laboratory investigations.

The aspirate from the abscess was subjected to Acid fast staining using microscopy (Ziehl-Neelsen staining) that revealed Acid Fast Bacilli (2+). In addition (Cartridge Based Nucleic Acid Amplification Test) confirmed the presence of M. tuberculosis complex sensitive to Rifampicin. J Microbiol Infect Dis 2020; 10(4): 234-236.
\end{abstract}

Keywords: M. tuberculosis complex, CB-NAAT, Abscess, Acid-fast bacilli

\section{INTRODUCTION}

Abscess formation at the site of vaccination occur when the vaccines are not given under proper aseptic conditions or when given by untrained staff. Most of the infections are caused by bacterial pathogens which include Staphylococcus aureus, Escherichia coli, Klebsiella pneumonia, Pseudomonas spp [1]. Rarely rapid growing nontuberculous mycobacteria (NTM) and M. bovis are also implicated due to contaminated needles or probable use of tap water, the common reservoir of $M$. chelonae and $M$. fortuitum [1-4]. Mycobacterium tuberculosis causing abscess formation at vaccination site is very rare and has been reported by few authors [1-3]. The diagnosis of such infections may be very difficult and challenging.

Intramuscular administration of vaccine contributes up to $21.5 \%$ of abscess formation [4]. Most of cases are due to use of a contaminated needle/ syringe and lack of aseptic measures while administration [5]. IM injections need proper aseptic precautions and appropriate administration technique to prevent rare but serious complications.
We report a rare case of tubercular soft tissue cold abscess formation following intramuscular vaccination. This work was given clearance by the ethical committee and written informed consent was also obtained from the child's parents.

\section{CASE REPORT}

A healthy eight months old male child (birth weight $3 \mathrm{~kg}$ ) developed induration and erythema at the site of an injection on anterolateral part of right thigh. The lesion appeared first time, five days following his third dose of intramuscular Hepatitis B vaccine which was administered at some private hospital. The previous vaccinations including the hepatitis B and BCG at birth followed by DPT, Hepatitis B second dose was tolerated well without any post-vaccination complications.

Clinical examination showed erythematous painless swelling of $2 \times 2.5 \mathrm{~cm}$ size, over anterolateral aspect of right thigh. It was firm, non-tender with normal body temperature and a sinus was observed. Movement of legs were not restricted. The child was afebrile and Vital signs were normal. Child was slightly irritable. The lesion had not responded to empirical antibiotic

Correspondence: Dr. Nahid Nehvi, Assistant Professor, Department of Microbiology, Government Medical College, Karan

Nagar, Srinagar, Jammu-Kashmir, India

Email: drnahidnahvi@gmail.com

Received: 13 June 2019 Accepted: 28 July 2020

Copyright (C JMID / Journal of Microbiology and Infectious Diseases 2020, All rights reserved 
therapy. There was no history of fever, cough, or weight loss. There was no history of contact with a known case of pulmonary tuberculosis in the family.

Fine needle aspiration cytology of abscess showed granulomatous inflammation. The serology was negative for HIV infection and aerobic bacterial culture was sterile. The aspirate was sent to Mycobacteriology laboratory for further investigations. The following tests were performed and the tests results were found positive for Mycobacterium tuberculosis in the Mycobacteriology laboratory: (1) Ziehl-Neelsen staining: positive for acid-fast bacilli (2+). (2) CB-NAAT: GeneXpert MTB/RIF assay (Real time nested PCR, Cepheid) revealed MTB complex sensitive to Rifampicin.

(3) Culture on L J Media: growth was seen after three weeks (tough, rough and buff colonies). These were again confirmed by Gene Xpert and MPT 64 Ag which showed positive results.

No evidence of tuberculosis on clinical and radiographic examination was seen. Hematological parameters showed moderate elevation of lymphocyte count. Immunodeficiency disorders were ruled out.

The baby was put immediately on anti-tubercular treatment as per the RNTCP (Revised national tuberculosis control program) after CB- NAAT showed positive result. Intensive therapy with Isoniazid $10 \mathrm{mg} / \mathrm{kg}$, rifampicin $15 \mathrm{mg} / \mathrm{kg}$, pyrazinamide $35 \mathrm{mg} / \mathrm{kg}$ and ethambutol 20 $\mathrm{mg} / \mathrm{kg}$ daily for first 2 months was started followed by continuation therapy with Isoniazid, rifampicin and ethambutol daily for 4 months was started one-month post abscess formation. Follow up after two months of intensive phase showed absence of abscess.

ATT was given for total period of 6 months. ATT regime was well tolerated by the infant.

\section{DISCUSSION}

Mycobacterium tuberculosis is a rare but important cause of cold abscess following injections and CB-NAAT is the most sensitive rapid test by which the infection can be diagnosed at the earliest and should be the first test to be done as culture although is the gold standard but it takes time for growth and identification of $M$. tuberculosis which may delay the treatment.
In this case, tubercular abscess was microbiologically confirmed by positive AFB microscopy and GeneXpert MTB RIF (nested real time PCR).

Previous researchers have reported cases of tuberculosis cold abscess caused by Mycobacterium tuberculosis following DTP vaccination. A variable time period observed between vaccination and appearance of swelling of five days in Mishra et al and 21 days in Dixit et al. [2-6].

In our study the swelling appeared after 5 days following the third booster dose of Hepatitis B vaccination.

The use of contaminated syringe or improper vaccination may be the cause of abscess. This occurs due to direct inoculation of injection site with coughed up tuberculosis bacilli by infected nursing staff, needle introducing tubercle bacilli from skin surface to injured tissue resulting in cold abscess due to $M$. tuberculosis have been reported [2,5,7-9].

A Similar study by Shah et al. describes cases of tubercular thigh abscess in an infant $\leq 6$ months old following hepatitis B vaccine. Granulomatous changes along with caseous necrosis were reported on histopathological examination [9].

It may be hypothesized that the intramuscular injections damage the local tissue in some way so that organisms, probably torpid somewhere else, lodge in these areas of decreased resistance. However, the exact mode of acquisition of infection remains unknown. The use of contaminated multidose vials, contaminated syringes and lack of aseptic precautions at the time of vaccination are some predisposing factors.

Also, the hematogenous spread of Tubercle bacilli from primary site (somewhere else in body) may get lodged at injection site and multiply to form an abscess. Such spread of tubercle bacilli from primary focus may take one week to three months and last for a very short time [1].

As conclusion, M. tuberculosis is an important, though rare cause of abscess even in an immunocompetent infant. High degree of clinical 
suspicion is required for early diagnosis and complete treatment for better outcome.

\section{ACKNOWLEDGMENTS}

Conflict of interest: The authors declare no personal or financial conflict of interest.

Financial Disclosure: No financial support was received.

\section{REFERENCES}

1. Gupta $P$, Menon PSN, Ramji $S$, et al. PG Textbook of Pediatrics: Infections and Systemic Disorders; 2018;2:1139-1147.

2. Mishra D, Mohta A, Arora P. Cold abscess of the thigh in an infant. Kathmandu Univ Med J. 2013; 41:86-87.

3. Agrawal A, Jain A. Tuberculosis Cold Abscess. Indian J Pediatr 2007; 74:771-773.

4. Gondal KM, Iqbal Y, Khan UA, et al. Frequency of Contributing Factorsin Intramuscular Injection Abscess. PJMHS 2014; 569-572.

5. Tan NWH, Pan WJ, Yunos HBM, et al. Postvaccination abscesses requiring surgical drainage in a tertiary childrens hospital in Singapore. J Pediatr Infect Dis 2010; 5:139-147.

6. Dixit $\mathrm{R}$, Dixit $\mathrm{K}$, Patil $\mathrm{CB}$, et al. Injection site abscess due to Mycobacteriology tuberculosis following DPT vaccinations. Indian J Tuberc 2014; 61:246-249.

7. Namshan M, Oda O, Zamakhshary $M$, et al. Bacillus Calmette Guerin related cold thigh abscess as an unusual cause of thigh swelling in infants following BCG vaccine administration: a case series. J Med Case Reports 2011; 5:472.

8. Verma S, Didel S. Cold abscess of thigh following inadvertent vaccination in an infant; Indian J Child Health 2016;3:76-78.

9. Shah A, Dash B. Primary Tubercular Abscess of Thigh in Infancy. Indian Pediatr 2015; 52:992.

10 Sharma J, Sharma T, Bhatt GC, et al. Isolated cold abscess of the thigh in an immunocompetent infant. Trop Doct 2014; 44:221-222. 